\title{
RESILIENCIA EN NIÑOS ENFERMOS CRÓNICOS: ASPECTOS TEÓRICOS ${ }^{1}$
}

\author{
Elisa Kern de Castro* \\ Bernardo Moreno-Jiménez
}

\begin{abstract}
RESUMEN. El presente trabajo examina cuestiones relacionadas a la enfermedad crónica, el desarrollo infantil y la resiliencia. La resiliencia es un concepto evolutivo y de la salud que se refiere a la capacidad de adaptación positiva de niños, jóvenes y adultos frente a circunstancias adversas y de riesgo, como es la enfermedad crónica pediátrica. Actualmente existen pocos estudios sobre el tema, pero las evidencias revelan que niños enfermos crónicos se adaptan de una manera superior a la que se esperaría. Por otro lado, algunos niños enfermos presentan desajustes emocionales y de conducta. El estudio de la resiliencia relacionada con la enfermedad crónica pediátrica significa un cambio de perspectiva en el campo de la salud ya que enfoca el desarrollo positivo del niño. La aplicación práctica de los conocimientos para promover la resiliencia todavía es un desafío para los profesionales de la salud.
\end{abstract}

Palabras-clave: niños, resiliencia, conducta frente a la enfermedad.

\section{RESILIÊNCIA EM CRIANÇAS COM DOENÇAS CRÔNICAS: ASPECTOS TEÓRICOS}

\begin{abstract}
RESUMO. O presente trabalho examina questões relacionadas a doenças crônicas, o desenvolvimento infantil e a resiliência. A resiliência é um conceito dos campos do desenvolvimento e da saúde que se refere à capacidade de adaptação positiva de crianças, jovens e adultos frente a circunstâncias adversas e de risco, como é a doença crônica pediátrica. Atualmente, existem poucos estudos sobre o tema, mas as evidências revelam que crianças enfermas cronicamente se adaptam melhor ao que se esperaria. Por outro lado, algumas crianças doentes apresentam problemas emocionais e comportamentais. O estudo da resiliência relacionada com a doença crônica pediátrica significa uma mudança de perspectiva no campo da saúde, uma vez que enfoca o desenvolvimento saudável da criança. A aplicação prática dos conhecimentos em resiliência é um desafio para os profissionais da saúde.
\end{abstract}

Palavras-chave: criança; resiliência; atitudes frente à doença.

\section{RESILIENCE IN CHILDREN WITH PHYSICAL CHRONIC ILLNESS: THEORETICAL ASPECTS}

\begin{abstract}
The present work examined some questions concerning the chronic disease, child development and resilience. Resilience is a developmental and health concept that refers to the children, adolescent and adults positive adaptation in the presence of adverse and risk circumstances, like is pediatric chronic disease. Currently there are few studies on the topic, but despite that, evidences showed that adaptation of chronic ill children is higher than would be expected. In contrast, some chronic ill children present emotional and behavior problems. Resilience related to pediatric chronic disease means a perspective change in health field because the focus is on child positive development. Practical applications of resilience findings are a challenge to health professionals.

Key words: Childhood, resilience, attitudes toward physical illness.

\footnotetext{
1 Apoio: CAPES.

* Psicóloga, Doctora. Professora adjunta del Programa de Pós-graduação em Psicologia de la Universidade do Vale do Rio dos Sinos-Unisinos.

\# Psicólogo, Profesor Titular y Director del Departamento de Psicología Biológica y de la Salud de la UAM-Espanha.
} 
Las enfermedades crónicas infantiles son un grupo heterogéneo de dolencias con distintas características. En su mayor parte, las enfermedades son progresivas, causan deterioro físico importante, perjudican el desarrollo del niño y requieren tratamientos dolorosos y largas hospitalizaciones. (Eiser, 1996; Garralda, 1994; Perrin \& Shonkoff, 2000). El tratamiento médico para esas enfermedades se ha desarrollado mucho en los últimos años, pero pocos estudios en Psicología han enfocado su impacto sobre el bienestar y salud mental del niño.

El objetivo del presente artículo es hacer una revisión teórica sobre el tema de la enfermedad crónica en la infancia en el contexto de la resiliencia. En la primera parte del trabajo se exploran algunas implicaciones de la enfermedad crónica para el desarrollo del niño. A continuación, se revisa el concepto de resiliencia en la infancia y se examina el concepto relacionado con la enfermedad crónica.

\section{LA ENFERMEDAD CRÓNICA Y EL DESARROLLO INFANTIL}

Niños sanos y niños enfermos tienen que superar las mismas tareas evolutivas, pero para estos últimos es mucho más complicado debido a la influencia de los aspectos fisiológicos de la propia enfermedad, tratamiento, hospitalizaciones, limitaciones $\mathrm{y}$ alteraciones en las relaciones familiares en su vida (Midence, 1994). Aley (2002) refiere que el objetivo del trabajo de los profesionales de salud es ayudar al niño enfermo a tener un desarrollo razonable dentro de las limitaciones impuestas por su condición y no sólo atender a su enfermedad.

Es fundamental que en los estudios con niños enfermos crónicos se comprendan los procesos evolutivos que caracterizan el período de la infancia y adolescencia en sus aspectos físicos, emocionales, cognitivos y sociales (Midence, 1994). Para comprender las conductas del niño frente a su enfermedad se debe identificar en qué fase del desarrollo se encuentra, y para eso no sólo la edad cronológica es importante, sino también las tareas evolutivas que haya superado (Pedreira, 1998).

Las habituales experiencias de dolor, daño orgánico y tratamiento intrusivo provocan cambios y alteraciones en la representación del cuerpo de niños y adolescentes enfermos (Blum-Gordillo, GordilloPaniagua \& Garcia-Lozano, 2003). Esos cambios corporales y el sufrimiento psíquico pueden generar sentimientos de inferioridad frente a las demás personas (el niño se siente "distinto" por estar enfermo), tristeza y vergüenza, con consecuencias para su autoconcepto y autoestima.

\section{RESILIENCIA}

El proceso de desarrollo por sí mismo constituye un modelo de adaptación a través del tiempo (Yates, Egeland \& Sroufe, 2003). Por eso, los niveles anteriores de adaptación son solamente probabilísticos, y no determinísticos, del funcionamiento posterior. La competencia en un cierto período evolutivo da al niño los fundamentos para seguir con su desarrollo en las etapas subsecuentes. Por otro lado, la inadaptación en un período evolutivo puede comprometer la capacidad del niño a un desarrollo posterior. Sin embargo, la naturaleza probabilística de las asociaciones entre sucesivos niveles de adaptación lleva a la heterogeneidad en los modelos evolutivos.

Por todo ello, el proceso adaptativo del niño nos lleva al concepto de resiliencia. El estudio de la resiliencia se refiere a cómo los niños y jóvenes se desarrollan cuando tienen que confrontar circunstancias adversas, valorizando los aspectos positivos de la adaptación (Masten \& Powell, 2003; Pesce, Assis, Santos \& Oliveira, 2004; Pinheiro, 2004; Stein, Fonagy, Ferguson \& Wisman, 2000). Desde un punto de vista evolutivo, los resultados adaptativos del desarrollo derivan de los intercambios transaccionales entre el niño y su ambiente, así como de su historia evolutiva (Yates \& cols., 2003). Dentro de esa perspectiva, la resiliencia es un concepto evolutivo y de la salud que caracteriza los procesos dinámicos que facilitan la organización e integración de la experiencia en modos de funcionamiento adaptativos. Ese concepto reafirma la capacidad del ser humano de superar situaciones potencialmente traumáticas transponiendo el determinismo y dando la posibilidad de esperanza de desarrollo adaptativo incluso en situaciones $o$ escenarios marcadamente conflictivos (Junqueira \& Deslandes, 2003). Por eso, Pesce y cols. (2004) afirman que la resiliencia no nace con el sujeto, sino que es adquirida con su desarrollo.

La adaptación positiva se refiere a la adaptación sustancialmente superior a la que se esperaría dada la exposición a circunstancias de riesgo (Yates \& Zelazo, 2003). Para definir la resiliencia hay que atender a la seriedad de los riesgos considerados: para niños que pasaron por serios traumas es totalmente apropiado considerar la disminución de riesgo o simplemente la ausencia de diagnóstico psicopatológico. En otros casos, en los que la situación que ha pasado el niño es conflictiva, pero transitoria y no traumática, la 
resiliencia puede ser valorada con relación a la madurez adquirida y la adaptación del niño en su vida diaria. Con eso, de acuerdo con Pesce y cols. (2004), la resiliencia traduce conceptualmente la posibilidad de superación en un sentido interactivo, sin que necesariamente represente la eliminación del problema, sino la aparición de un nuevo contexto de significado del problema. En el lado opuesto está la vulnerabilidad, que es la predisposición individual del individuo para desarrollar formas variadas de psicopatología o conductas no eficaces, o la susceptibilidad de un resultado negativo en su desarrollo.

Ese concepto ya aparecía en la Teoría del Apego, de Bowlby, al atribuir la aparición de la resiliencia a las primeras experiencias del niño con la madre, el padre y otros, ya que las experiencias positivas contribuyen a la formación de personalidades saludables y resilientes (Bowlby, 1976; Pinheiro, 2004). Sin embargo, el punto de vista adoptado en la actualidad no considera la resiliencia como un atributo de personalidad. Para Stein y cols. (2000), la relación entre resiliencia y períodos críticos del desarrollo deben ser examinados, porque aunque es sabido que los primeros años de vida son cruciales para el establecimiento de la salud o psicopatología, hoy también es conocido que el cerebro es extremamente flexible y que su desarrollo continúa más allá de los tres años de edad.

La literatura sobre resiliencia sugiere que existen algunas características fundamentales de los sistemas en funcionamiento que aumentan significativamente la adaptación ante distintos estresores y situaciones (Masten \& Powell, 2003). Esos sistemas son versátiles y responsivos a una gran variedad de cambios, tanto normativos como no normativos. Sus formas de actuación pueden ser diferentes en función del desarrollo del niño y de su historia, de las situaciones y el contexto. Por ejemplo, algunos padres de niños que han pasado por serios traumas pueden creer en su capacidad de superación, ayudándoles a una adaptación positiva a pesar de las adversidades.

Es importante tener claro que identificar la resiliencia desde un diagnóstico explícito o implícito no significa que se ha descrito la totalidad de la persona o las características de su vida (Masten \& Powell, 2003). Técnicamente, considerar a una persona resiliente es impropio de la terminología diagnóstica porque resiliencia es la descripción de un modelo general. Sería más adecuado decir que 'esa persona posee características de resiliencia', que pueden ser transitorias y específicas a algunos contextos.
Es crucial identificar los factores de riesgo y protección implicados en la resiliencia para utilizarla de una manera terapéutica (Place, Reynolds, Cousins \& O'Neill, 2002). Los factores de protección (como autoestima, apoyo social, género y relaciones con otras personas) facilitan el proceso individual de percibir y afrontar el riesgo (Pesce \& cols., 2004). Los conceptos de riesgo y protección no pueden ser considerados homogéneos. Un determinado evento de vida adverso puede ser considerado factor de riesgo para la adaptación de un individuo pero puede no tener el mismo efecto para otra persona. Por otra parte, a pesar de que los factores de protección tengan una relación más directa con la resiliencia, deben ser entendidos tanto separadamente como en conjunto con otros factores y con el propio riesgo.

Desarrollar la resiliencia no significa que la persona haya superado todas sus experiencias traumáticas (Junqueira \& Deslandes, 2003). La resiliencia no es un proceso lineal, pues un individuo puede salir bien delante de una determinada situación pero, posteriormente, no hacerlo bien frente a otra.

En el caso de los niños, han sido identificados un gran número de factores de protección ante las adversidades que han sido agrupados en tres áreas: individuales, de sus familias y de su comunidad (Masten \& Powell, 2003; Place \& cols., 2002). Algunos factores de protección individuales son alta autoestima y autoconcepto positivo, habilidad de autoreflexión, capacidad de pensar y actuar de manera independiente, habilidades de solución de problemas, adaptabilidad social, etc. Entre los factores de protección familiares se destacan la cohesión y apoyo y el sentimiento de un vínculo afectivo fuerte con los progenitores, y entre los factores de protección de la comunidad sobresalen la influencia de amigos y las relaciones íntimas con adultos competentes que le sirvan de modelo positivo, así como la conexión con organizaciones pro sociales (clubes, grupos), la calidad del entorno (seguridad pública, centros recreativos...) y la calidad de los servicios sociales y de atención a la salud.

Como factores de riesgo son considerados aquellos obstáculos que aumentan la vulnerabilidad en el desarrollo del niño (Masten \& Powell, 2003; Pesce \& cols., 2004). Algunos factores de riesgo crónicos o agudos que pueden perjudicar el desarrollo de niños y adolescentes son prematuridad al nacer, pobreza, problemas familiares, violencia, enfermedad, pérdidas, maltrato, guerras, etc. Tales factores no pueden ser considerados aislados, sino que hay que considerar su interacción y proceso de desarrollo. Estos 
contextos son establecidos como factores de riesgo para el desarrollo porque hay fuertes evidencias de que predicen altas tasas de resultados negativos $o$ indeseables para la vida del individuo. Estudios antiguos enfocaban la atención en sólo un factor de riesgo, sin embargo, es notorio que esos factores de riesgo co-ocurren con otros factores de riesgo, usualmente sincronizados con una secuencia de experiencias estresantes y no sólo de un evento único. Como resultado, muchos investigadores han puesto su atención en riesgos acumulativos.

Los estudios sobre resiliencia utilizan distintos abordajes en el intento de identificar los factores asociados con una mejor adaptación en niños de riesgo (Masten \& Powell, 2003). Dos son los abordajes principales que caracterizan la investigación sobre resiliencia: el abordaje enfocado en una variable y el enfocado en la persona. El abordaje enfocado en una variable examina las relaciones entre competencia, adversidad, y factores potencialmente protectores relacionados con las variables que describen diferencias entre niños individuales y la naturaleza de esas relaciones e interacciones con el mundo en que viven. Esos modelos son importantes no sólo para testar hipótesis de factores protectores, sino también porque pueden servir como modelos de intervención. En cambio, el abordaje enfocado en la persona busca identificar personas que cumplen criterios de resiliencia, cuyas vidas e atributos han sido examinados por investigadores, particularmente en comparación con individuos poco adaptados que tienen niveles similares de riesgo y adversidad pero que tienen resultados evidentemente distintos. Ese abordaje es menos sensible que el anterior para identificar procesos relacionados con dominios específicos de competencia, pero tal vez pueda mostrar mejor los modelos de resiliencia que ocurren realmente. Los estudios de caso, por ejemplo, pueden ofrecer ideas sobre resiliencia, pero su generalización es problemática.

En los estudios empíricos sobre resiliencia, la primera tarea complicada es la operacionalización del término, y asumen que la resiliencia nunca puede ser medida directamente y que es inferida basándose en medidas directas: riesgo y adaptación positiva (Yates \& Zelazo, 2003). Por eso, es frecuente la utilización de varios instrumentos de naturaleza distinta debido a la dificultad de evaluar la resiliencia a través de un único parámetro de medida (Pesce \& cols., 2004).

\section{RESILIENCIA EN LA ENFERMEDAD CRÓNICA INFANTIL}

La enfermedad crónica infantil y todo lo que conlleva (tratamiento, hospitalizaciones, etc.) puede ser considerada un factor de riesgo para el desarrollo del niño. En el contexto de la resiliencia, pocos estudios han intentado comprender la adaptación del niño frente a esta situación adversa.

Eventos negativos agudos pueden provocar efectos negativos en un corto plazo pero no siempre a largo plazo, y un ejemplo claro es la hospitalización infantil (Pesce \& cols., 2004). Sin embargo, repetidas hospitalizaciones podrían estar asociadas a adversidades psicosociales.

De acuerdo con Gartstein, Noll y Vannatta (2000), muchos niños que sufren de enfermedades crónicas demuestran capacidad de superación y adaptación positiva a la experiencia. La disminución de riesgos de problemas de conducta externalizante en esos niños puede estar asociada al impacto de la enfermedad en su funcionamiento psicológico (frecuentes episodios sintomáticos, disminución de la competencia física), o con el efecto de la enfermedad en los padres, especialmente relacionado con el nivel de supervisión que tienen de las actividades del niño. Wallander, Varni y Babani (1989) han propuesto un modelo conceptual sobre la enfermedad crónica en la infancia que ha sido utilizado y complementado por otros autores como Boekaerts y Röder (1999) y Bradford (1997). Para esos autores, la adaptación del niño a la enfermedad crónica está determinada por la interacción entre los factores de riesgo y protección. Tres factores son esenciales en los factores de riesgo: variables de la enfermedad (gravedad, visibilidad y problemas médicos asociados), nivel de funcionamiento independiente del niño, y estresores psicosociales que se relacionan con la enfermedad. Entre los factores de protección están las variables intrapersonales (temperamento, habilidad para resolver problemas, etc.), ecología social (ambiente familiar, apoyo social, recursos de la comunidad) y habilidades en el procesamiento del estrés (evaluación cognitiva y estrategias de afrontamiento). Las investigaciones en el ámbito psicosocial en los últimos años se han enfocados en los factores protectores que están relacionados con los pacientes que sufren de alguna enfermedad y que funcionan como mediadores para la resiliencia (Serrano-Ikos \& Lask, 2003).

La literatura científica sobre psicopatología y enfermedad crónica en la infancia apunta a un mayor riesgo de problemas emocionales/conductuales de diversos tipos para esa población, pero sólo una minoría de niños de hecho presenta problemas 
psicológicos (Bradford, 1997; Burke \& Elliot, 1999; Gartstein \& cols., 2000). Para los autores, los esfuerzos que se han hecho para entender las necesidades de salud mental de esos niños han sido problemáticas por limitaciones metodológicas: la mayoría de los estudios utilizan sólo informes de padres sobre las conductas del niño porque son medidas de fácil administración y puntuación y porque muchos investigadores consideran la información obtenida de los padres como la más válida y fiable, pero es sabido que la opinión de los padres tiene sus sesgos, especialmente por el hecho de que muchos progenitores de niños enfermos crónicos son sobreprotectores (Gartstein \& cols., 2000). Aunque el aumento del control de las conductas de los niños por parte de los padres disminuya los riesgos de problemas de agresión, los mensajes transmitidos al niño con esa actitud son de inutilidad, incapacidad para cuidarse de sí mismo e inseguridad.

Todavía no están claros los factores y los mecanismos que predisponen a una parte de los niños enfermos crónicos a tener desajustes emocionales o de conducta. Bennet (1994) considera que los trastornos psicológicos de niños enfermos se deben, en parte, a sus pocas oportunidades de socialización, a su extrema dependencia a sus padres y al equipo de salud y a sus limitaciones físicas. Evidencias recientes apuntan a una fuerte asociación entre número de hospitalizaciones en el primer año de vida y la presencia de problemas de conducta (Anselmi, Piccinini, Barros \& Lopes, 2004). Otros factores, además de la salud física, pueden influenciar las respuestas del niño a la enfermedad, como el funcionamiento familiar, las pocas actividades sociales y la dependencia exagerada a adultos (Silver, Stein \& Dadds., 1996). Es muy difícil de predecir la aparición de psicopatología en la adolescencia y edad adulta solamente a partir de características de la infancia, ya que los niños en situación de riesgo pueden encontrar un entorno ambiental que actúe como factor de compensación, o porque tienen un temperamento que les permite afrontar las situaciones adversas de manera eficaz. (Giráldez, 2003).

Trabajar por un enfoque positivo relacionado con la promoción de la salud es posible según Junqueira y Deslandes (2003). Reafirmar la importancia de la autoestima en niños y adolescentes, divulgar sus derechos, respetar su propio cuerpo, reforzar el diálogo y la tolerancia en la familia son trabajos de prevención primaria que pueden ser realizados con niños $\mathrm{y}$ adolescentes que pasan por adversidades de distintos tipos. No obstante, la promoción de la resiliencia no sustituye las acciones del Estado en las cuestiones referentes a las situaciones de riesgo a la salud y a las que interfieren negativamente en la calidad de vida. Promover la resiliencia requiere también políticas relacionadas con la mejora de situaciones adversas y de asistencia a las necesidades de las personas, de forma que a la persona le resulte más fácil desarrollar características resilientes si existen condiciones personales y ambientales favorables. Finalmente, una lectura crítica del concepto de resiliencia ilustra sobre los posibles aspectos ideológicos debido a que la capacidad de adaptación del individuo al ambiente social puede ser interpretada como conformidad a las reglas establecidas. Al enfocarse las características de las personas en el trabajo con resiliencia es fundamental contextualizar el aspecto de las interacciones y cuidados al niño y adolescente, cuidando de no caer en un planteamiento simplemente individualista.

\section{CONSIDERACIONES FINALES}

La diversidad de resultados en el desarrollo de niños que sufren enfermedades crónicas es enorme. Depende, en gran medida, de la propia condición física y limitaciones de la enfermedad que sufren, pero también de los recursos personales, familiares y sociales de que disponen.

El estudio de la resiliencia es relativamente reciente en Psicología, y más aún cuando se aplica a la enfermedad crónica como un factor de riesgo para el desarrollo. Significa un cambio importante de perspectiva, ya que enfoca el desarrollo positivo, la posibilidad de superación y adaptación ante la enfermedad.

Sin duda, los programas de salud mental en contextos comunitarios y hospitalarios pueden ayudar a los niños enfermos crónicos a una mejor adaptación a su dolencia. Sin embargo, el desarrollo de la resiliencia todavía es un desafío para los investigadores y para una aplicación práctica de los conocimientos ya adquiridos. ¿Existe alguna manera de ayudar a los niños a poseer características de resiliencia? ¿En qué medida la familia puede ayudar al niño enfermo a desarrollar la resiliencia frente a su molestia? ¿La superación de situaciones adversas que supone la resiliencia, como en la enfermedad crónica infantil, puede ser incorporada a otros contextos de situaciones adversas por el mismo individuo? ¿La capacidad de superación del niño frente a la enfermedad crónica es una característica que es añadida de forma permanente para toda su vida?

En definitiva, en el contexto de la enfermedad crónica, el abordaje de la resiliencia empieza ahora a 
tomar forma, pero hay mucho todavía por conocer. Es importante marcar que el cambio de enfoque de la enfermedad crónica infantil que asume el abordaje de la resiliencia no significa que se deje de mirar a los problemas que puedan causar tal situación (problemas emocionales, disfunciones familiares, etc.). Sin embargo, este reciente planteamiento contiene una visión optimista y esperanzadora para el desarrollo de los niños enfermos crónicos y que tienen que aprender a convivir con una molestia para toda su vida.

\section{REFERÊNCIAS}

Aley, K. E. (2002). Developmental approach to pediatric transplantation. Progress in Transplantation, 12(2), 86-91.

Anselmi, L., Piccinini, C. A., Barros, F. \& Lopes, R. (2004). Psychosocial determinants of behaviour problems in Brazilian preschool children. Journal of Child Psychology and Psychiatry, 45(4), 779-788.

Bennet, D. S. (1994). Depression among children with chronic medical problems: A meta-analysis. Journal of Pediatric Psychology, 19(2), 149-169.

Blum-Gordillo, B., Gordillo-Paniagua, G. \& Garcia-Lozano, D. (2003, $2^{a}$ ed.). Psiconefrología. En G. Gordillo-Paniagua, R. A. Y de la Cruz \& J. Exeni (Orgs.), Nefrología Pediátrica (pp 579-596). Madrid: Elsevier Science.

Boekaerts, M. \& Röder, I. (1999). Stress, coping and adjustment in children with a chronic disease: A review of the literature. Disability and Rehabilitation, 21(7), 311-337.

Bowlby, J. (1976). El vínculo afectivo. Apego y pérdida: Vol. 1 (I. Pardal, Trad.) Buenos Aires: Paidós. (Original publicado en 1969).

Bradford, R (1997). Children, families and chronic disease. London: Routledge.

Burke, P. \& Elliot, M. (1999). Depression in pediatric chronic illness: A diathesis-stress model. Psychosomatics, 40(1), 243249.

Eiser, C. (1996). Helping the child with chronic disease: Themes and directions. Clinical Child Psychology and Psychiatry, 1(4), 551-561.

Garralda, M. E. (1994). Chronic Physical Illness and Emotional Disorder in Childhood. British Journal of Psychiatry, 164, 810.

Gartstein, M. A., Noll, R. B. \& Vannatta, K. (2000). Childhood aggression and chronic illness: Possible protective mechanisms. Journal of Applied Developmental Psychology, 21(3), 315-333.

Giráldez, S. L. (2003). La psicopatología de la infancia y la adolescencia: consideraciones básicas para su estudio. Papeles del Psicólogo, 85, 19-28.
Junqueira, M. F. P. \& Deslandes, S. F. (2003). Resiliência e maustratos à criança. Cadernos de Saúde Pública, 19(1), 227-235.

Masten, A. S. \& Powell, J. L. (2003). A resilience framework for research, policy, and practice. In S. S. Luthar (Ed.), Resilience and Vulnerability: Adaptation in the context of childhood adversities (pp. 1-29). Cambridge: University Press.

Midence, K. (1994). The effects of chronic illness on children and their families: An overview. Genetic, Social \& General Psychology Monographs, 120(3), 311-316.

Pedreira Massa, J. L. (1998). El niño enfermo crónico: aspectos psicosomáticos y relacionales. Psiquis, 19(3), 89-96.

Perrin, J. M. \& Shonkoff, J. P. (2000). Developmental disabilities and chronic illness: An overview. In R. E. Behrman, R. M. Kliegman \& H. B. Jenson (Eds.), Nelson Textbook of Pediatrics (pp. 452-464). Philadelphia: W. B. Saunders.

Pesce, R., Assis, S. G., Santos, N. \& Oliveira, R. (2004). Risco e proteção: em busca de um equilíbrio promotor de resiliência. Psicologia: Teoria e Pesquisa, 20(2), 135-143.

Pinheiro, D. P. (2004). A resiliência em discussão. Psicologia em Estudo, 9(1), 67-75.

Place, M., Reynolds, J., Cousins, A. \& O'Neill, S. (2002). Developing a resilience package for vulnerable children. Child and Adolescent Mental Health, 7(4), 162-167.

Serrano-Ikos, E. \& Lask, B. (2003). The psychosocial correlates of transplant survival. Journal of Cystic Fibrosis, 2, 49-54.

Silver, E. J., Stein, R. E. K. \& Dadds, M. R. (1996). Moderating effects of family structure on the relationship between physical and mental health in urban children with chronic illness. Journal of Pediatric Psychology, 21(1), 43-56.

Stein, H., Fonagy, P., Ferguson, K. S. \& Wisman, M. (2000). Lives through time: An ideographic approach to the study of resilience. Bulletin of the Menninger Clinic, 64(2), 281-305.

Wallander, J. L., Varni, J.W. \& Babani, L. (1989). Family resources of resistance factors for psychological maladjustment in chronically ill and handicapped children. Journal of Pediatric Psychology, 14(2), 157-173.

Yates, T. M., Egeland, B. L. \& Sroufe, A. (2003). Rethinking resilience: A developmental process perspective. In S. S. Luthar (Ed.), Resilience and Vulnerability: Adaptation in the context of childhood adversities (pp. 243-266). Cambridge: University.

Yates, T. M. \& Zelazo, L. B. (2003). Research on resilience: An integrative review. In S. S. Luthar (Ed.), Resilience and Vulnerability: Adaptation in the context of childhood adversities (pp. 510-549). Cambridge: University.

Recebido em 04/05/2006 Aceito em 14/07/2006

Dirección para correspondencia: Elisa Kern de Castro. Centro de Ciências da Saúde, sala 2A 112, Universidade do Vale do Rio dos Sinos, Av. Unisinos, 950, CEP 93022-000, São Leopoldo-RS. E-mail: elisakc@unisinos.br 